УДК 004.94, 519.63

DOI 10.18413/2687-0932-2020-47-3-610-622

\title{
Компьютерная модель, примеры анализа влияния ландшафтно-метеорологических факторов на динамику низовых лесных пожаров
}

\author{
Д.В. Баровик, В.Б. Таранчук \\ Белорусский государственный университет, \\ Беларусь, 220030, г. Минск, пр. Независимости, 4 \\ E-mail:BarovikD@gmail.com, taranchuk@bsu.by
}

\begin{abstract}
Аннотация
Обсуждаются результаты разработки и адаптации компьютерной модели для прогнозирования распространения низовых лесных пожаров, математического описания в принятом приближении соответствующих физико-химических процессов с уточнениями включаемых в уравнения взаимосогласованных определяющих функций и коэффициентов. Платформа разработки компьютерной модели, проведения расчетов, формирования базы данных с результатами вычислений - система компьютерной алгебры Wolfram Mathematica. Представлены данные вычислительных экспериментов по изучению возможных сценариев распространения зоны горения вблизи противопожарных разрывов во фронте, тыле и флангах пожара. Выявлены, иллюстрируются многомерной графикой несколько качественных особенностей в эволюции температурного фронта, его распространении на площади лесного массива при наличии полян различной формы, в том числе, с демонстрацией влияния равновесной скорости ветра в пологе леса. Подобраны и поясняются представительными примерами возможные варианты динамики фронта пожара в направлениях по и против ветра.
\end{abstract}

Ключевые слова: низовой лесной пожар, математическая модель, динамика фронта, противопожарный разрыв, форма поляны, скорость ветра, программный комплекс.

Для цитирования: Баровик Д.В., Таранчук В.Б. 2020. Компьютерная модель, примеры анализа влияния ландшафтно-метеорологических факторов на динамику низовых лесных пожаров. Экономика. Информатика. 47 (3): 610-622. DOI 10.18413/2687-0932-2020-47-3-610-622.

\section{Computer model, examples of analysis of landscape and meteorological factors affecting the dynamics of surface forest fires}

\author{
D.V. Barovik, V.B. Taranchuk \\ Belarusian State University, 4 Nezavisimosti Ave, Minsk, 220030, Belarus \\ E-mail: BarovikD@gmail.com, taranchuk@bsu.by
}

\begin{abstract}
The article discusses the results of development and instrumental setup of a computer model for predicting the spread of surface forest fires, mathematical description in the accepted approximation of the corresponding physical and chemical processes with refinements of mutually agreed defining functions and coefficients included in the equations. The Wolfram Mathematica computer algebra system is used as a platform for developing the computer model, performing calculations, and creating a database with the outcomes of computations. The results of numerical experiments investigating possible scenarios how fire zone spreads in different directions and its behavior near fuelbreaks are presented. Several qualitative differences in geometry and dynamics of temperature density maps are determined and illustrated by multidimensional graphics for various shapes of fuelbreaks, including the demonstration of the influence of the equilibrium wind speed in the forest canopy on the fire spread over the territory. Possible variants of the fire front movement in the direction of wind velocity and against it are identified and explained using representative examples.
\end{abstract}


Keywords: surface forest fire, wildland fire, wildfires, mathematical model, fire front dynamics, fuelbreak shape, wind velocity, software.

For citation: Barovik D.V., Taranchuk V.B. 2020. Computer model, examples of analysis of landscape and meteorological factors affecting the dynamics of surface forest fires. Economics. Information technologies. 47 (3): 610-622 (in Russian). DOI 10.18413/2687-0932-2020-47-3-610-622.

\section{Введение}

Лесные массивы, являющиеся одним из основных типов растительного покрова Земли, подвержены влиянию целого ряда различных природных и техногенных факторов. Наибольший экологический и экономический ущерб на состояние лесных экосистем оказывают пожары [Dvornik et al., 2018; Чешко, 2019]. Примером являются пожары в Австралии, начавшиеся в сентябре 2019 года. По подсчетам Всемирного фонда дикой природы [wwf.org.ua, 2020], в них погибло более миллиарда животных. По мнению специалистов центра Moody's Analytics, по состоянию на начало 2020 года экономический ущерб превысит 4.4 миллиарда долларов США [theguardian.com, 2020].

На земном шаре существуют целые регионы, в которых лесные пожары происходят с регулярной периодичностью, и при этом не возрастает успешность в их предотвращении и тушении. Разработки математических моделей лесных пожаров начались с середины прошлого века в США и активно продолжаются во всем мире в настоящее время [Волокитина и др., 2020]. Прорыв в этих исследованиях позволит решать значительное число задач практического и научного характера. Обзор научных публикаций указывает как на определенные достижения, так и на ряд нерешенных вопросов [Perminov, Goudov, 2017; Antonov et al., 2018; Гладской и др., 2019; Frangieh et al., 2020]: недостаточная обоснованность принимаемых в моделях уравнений и их коэффициентов для описаний кинетики физикохимических превращений и реакций [Барановский, Захаревич, 2019; Ласута, Гоман, 2019]; сложность выбора адекватных моделей турбулентности в газовой фазе [Сыродой, Кузнецов, 2018]; незначительное число аналитических решений и масштабных натурных экспериментов, которые можно брать за эталон для верификации моделей. Наблюдается пробел между слишком упрощенными моделями, дающими прогнозы с большой погрешностью, и моделями с таким чрезмерным количеством параметров, что разработка быстродействующих численных методов для их решения становится самостоятельной проблемой [Баровик, Таранчук, 2011е].

Обзор научных статей и монографий позволяет условно разделить модели лесных пожаров по их математической «начинке» на следующие три группы [Баровик, Таранчук, 2011c]: теоретические, статистические [Гоман и др., 2011] и полуэмпирические [Баровик, Таранчук, 2011d, f].

При создании статистических (эмпирических) моделей основой являются: сбор и систематизация статистических данных о наблюдавшихся в прошлом скоростях распространения лесных пожаров в зависимости от выбранных и запротоколированных основных параметров (скорость ветра, температура окружающей среды, вид лесных насаждений и др.); статистическая обработка полученных результатов, определение коэффициентов корреляции между выбранными переменным и скоростью распространения огня. Прогноз в таких моделях дается с определенной вероятностью, полученные соотношения могут показать неудовлетворительные результаты в ситуациях, отличных от тех, в которых были собраны эмпирические данные [Гоман и др., 2011].

Полуэмпирические модели строятся на основе предположений о виде формул, описывающих скорости распространения пожара. Например, модель Ротермела предполагает, что низовой пожар распространяется в виде вытянутого по направлению ветра эллипса. Для связи между входными параметрами (угол рельефа, сила ветра, количество горючей растительности, ее влажность и т. п.) и скоростью фронта пожара привлекаются общие физические законы сохранения массы и энергии. Эти законы упрощаются путем замены в 
выражениях целого ряда членов на коэффициенты, подбор которых для конкретных типов лесных насаждений и климатических условий производится путем обобщения экспериментальной информации. Практика показывает, что такие полуэмпирические модели более точны по сравнению со статистическими (эмпирическими) моделями. В то же время такие модели значительно проще в программной реализации по сравнению с теоретическими [Баровик, Таранчук, 2011d, f].

Для построения теоретических (математических) моделей привлекаются фундаментальные законы тепломассопереноса, горения, газовой динамики, химических реакций и др. Записываются такие модели в виде системы дифференциальных уравнений в частных производных. Применение численных методов на высокопроизводительных компьютерах является основным инструментом для их решения. Вознаграждением за сложность таких моделей является то, что именно теоретические модели позволяют отвечать на широкий круг вопросов. Они дают возможность не только качественно, но и количественно исследовать динамику всего спектра величин в зоне пожара, тестировать различные методы по предотвращению, возникновению всех видов пожаров (низовых, верховых, пятнистых), развитию и их тушению с учетом конкретных климатических и территориальных факторов.

Профессором Гришиным А.М. [1992] была создана наиболее общая теоретическая модель распространения лесных и торфяных пожаров. Целый ряд исследователей [Perminov, Goudov, 2017; Кулешов, Мышецкая, 2019; Марзаева, 2019], включая авторов данной работы, берут модель Гришина за основу и модифицируют ее [Баровик, Таранчук, 2010b] для целей практического использования [Barovik, Taranchuk, 2010a].

\section{Математическое описание процесса распространения низовых лесных пожаров}

Ниже приводится двумерная (осредненная по высоте полога леса) математическая модель распространения лесных пожаров, позволяющая рассчитывать распределения по пространству и эволюцию по времени следующих величин: $T$ - измеряемая в Кельвинах температура лесного массива как сплошной многофазной реагирующей среды; $\varphi_{j}, j=1,2,3,4$ - объемные доли компонент лесного горючего материала (ЛГМ), где через $\varphi_{1}$ обозначено сухое органическое вещество ЛГМ, $\varphi_{2}$ - содержащаяся в древесине (растительности) вода в связанной и свободной формах, $\varphi_{3}-$ коксик (древесный уголь), являющийся продуктом пиролиза ЛГМ в условиях недостатка кислорода, $\varphi_{4}-$ зола (негорючая минеральная часть ЛГМ); $c_{v}, v=1,2,3$ - массовые концентрации компонентов газовой фазы, где $c_{1}$ - кислород, $c_{2}$ - горючие газы, возникающие в процессе термического разложения, $c_{3}$ - смесь остальных негорючих газов (водяной пар, как результат сушки; углекислый газ, выделяющийся при догорании коксика и окислении горючих газов; инертные компоненты воздушной смеси и продуктов реакций пиролиза и горения).

Предложенное ниже математическое описание позволяет учитывать следующие физико-химические процессы: теплоподвод, обусловленный конвекцией, теплопроводностью и радиационным излучением; нагрев ЛГМ; испарение воды из них (сушка); разложение сухого органического вещества ЛГМ (целлюлозы) на компоненты (газы, уголь, золу) под воздействием высокой температуры (пиролиз); горение газообразных и догорание твердых продуктов пиролиза. Детали упрощения модели, ее верификация, преобразования и получение уравнений, соображения о границах применимости, аппроксимирующая конечно-разностная схема и особенности возможной интерактивности при проведении расчетов приведены в статьях [Баровик, Таранчук, 2010с; Баровик, 2010].

В формулируемой начально-краевой задаче определяемыми функциями модели являются $T, \varphi_{1}, \varphi_{2}, \varphi_{3}, \varphi_{4}, c_{1}, c_{2}, c_{3}$. Эти функции зависят как от времени, так и от пространственных координат, и связаны соотношениями (1-16):

$$
\frac{\partial \varphi_{1}}{\partial t}=\Phi_{\varphi 1}\left(\varphi_{1}, T\right), \quad \frac{\partial \varphi_{2}}{\partial t}=\Phi_{\varphi 2}\left(\varphi_{2}, T\right), \quad \frac{\partial \varphi_{3}}{\partial t}=\Phi_{\varphi 3}\left(\varphi_{1}, \varphi_{3}, c_{1}, c_{2}, T\right), \quad \frac{\partial \varphi_{4}}{\partial t}=0,
$$




$$
\begin{gathered}
\frac{\partial c_{1}}{\partial t}+\left(V, g r a d c_{1}\right)-\frac{1}{\rho_{5}} \operatorname{div}\left(\rho_{5} D_{T} \operatorname{grad}_{1}\right)=\Phi_{c 1}\left(\varphi_{1}, \varphi_{2}, \varphi_{3}, c_{1}, c_{2}, T\right), \\
\frac{\partial c_{2}}{\partial t}+\left(V, g r a d c_{2}\right)-\frac{1}{\rho_{5}} \operatorname{div}\left(\rho_{5} D_{T} \operatorname{grad}_{2}\right)=\Phi_{c 2}\left(\varphi_{1}, \varphi_{2}, \varphi_{3}, c_{1}, c_{2}, T\right), \\
\frac{\partial T}{\partial t}+\frac{\rho_{5} c_{p 5}(V, \operatorname{grad} T)-\operatorname{div}\left(\lambda_{T} \operatorname{grad} T\right)}{\rho_{5} c_{p 5}+\sum_{j=1}^{4} \rho_{j} \varphi_{j} c_{p j}}=\Phi_{T}\left(\varphi_{1}, \varphi_{2}, \varphi_{3}, c_{1}, c_{2}, T\right) .
\end{gathered}
$$

Функции (правые части), записанных дифференциальных уравнений:

$$
\begin{gathered}
\Phi_{\varphi 1}\left(\varphi_{1}, T\right)=-\frac{R_{1}}{\rho_{1}}, \quad \Phi_{\varphi 2}\left(\varphi_{2}, T\right)=-\frac{R_{2}}{\rho_{2}}, \quad \Phi_{\varphi 3}\left(\varphi_{1}, \varphi_{3}, c_{1}, c_{2}, T\right)=\frac{\alpha_{c} R_{1}}{\rho_{3}}-\frac{M_{C}}{M_{1}} \frac{R_{3}}{\rho_{3}} \\
\Phi_{c 1}\left(\varphi_{1}, \varphi_{2}, \varphi_{3}, c_{1}, c_{2}, T\right)=\frac{1}{\rho_{5}}\left(R_{51}-c_{1} Q-\frac{\alpha}{c_{p 5} \Delta h}\left(c_{1}-c_{1 \infty}\right)\right) \\
\Phi_{c 2}\left(\varphi_{1}, \varphi_{2}, \varphi_{3}, c_{1}, c_{2}, T\right)=\frac{1}{\rho_{5}}\left(R_{52}-c_{2} Q-\frac{\alpha}{c_{p 5} \Delta h}\left(c_{2}-c_{2 \infty}\right)\right) \\
\Phi_{T}\left(\varphi_{1}, \varphi_{2}, \varphi_{3}, c_{1}, c_{2}, T\right)=-\frac{q_{2} R_{2}+q_{3} R_{3}+q_{5} R_{5}-\frac{\alpha}{\Delta h}\left(T-T_{\infty}\right)-4 \kappa_{R} \sigma T^{4}}{\rho_{5} c_{p 5}+\sum_{j=1}^{4} \rho_{j} \varphi_{j} c_{p j}}, \\
\sum_{v=1}^{3} c_{v}=1, \quad \rho_{5}=\frac{\rho_{\infty} T_{\infty}}{M_{\infty} T}\left(\sum_{v=1}^{3} \frac{c_{v}}{M_{v}}\right)^{-1}, \quad Q=\left(1-\alpha_{c}\right) R_{1}+R_{2}+\frac{M_{C}}{M_{1}} R_{3}, \\
R_{1}=k_{01} \rho_{1} \varphi_{1} \exp \left(-\frac{E_{1}}{R T}\right), R_{2}=k_{02} T^{-1 / 2} \rho_{2} \varphi_{2} \exp \left(-\frac{E_{2}}{R T}\right), R_{3}=k_{03} s_{\sigma} \varphi_{3} \rho_{5} c_{1} \exp \left(-\frac{E_{3}}{R T}\right), \\
R_{51}=-R_{3}-\frac{R_{5} M_{1}}{2 M_{2}}, R_{52}=\left(1-\alpha_{c}\right) v_{T} R_{1}-R_{5}, R_{5}=\rho_{5} \min \left(c_{2}, \frac{M_{2}}{2 M_{1}} c_{2}\right) k_{C O} \exp \left(-\frac{E_{C O}}{R T}\right)
\end{gathered}
$$

Используются обозначения: $t$ - время; рельефно-метеорологические характеристики задаются не возмущенной пожаром температурой $T_{\infty}$ окружающей среды, направлением и скоростью ветра $V$, высотой моделируемого слоя ЛГМ $\Delta h ; \rho_{j}, j=1,2,3,4$ - истинная плотность $\varphi_{j}$-ой компоненты ЛГМ; $\rho_{5}-$ плотность смеси газов (газовой фазы); $\rho_{\infty}-$ не возмущенная пожаром плотность воздуха; $R$ - универсальная газовая постоянная; константы $c_{1 \infty}$ И $c_{2 \infty}-$ массовые концентрации кислорода и горючих газов в невозмущенной атмосфере; $M_{1}, M_{2}, M_{3}, M_{\mathrm{C}}$ и $M_{\infty}-$ молекулярные массы кислорода, угарного газа, смеси негорючих газов, углерода (древесного угля) и не возмущенная пожаром молекулярная масса воздуха соответственно; $c_{p j}, j=1,2,3,4$ - теплоемкости $\varphi_{j}$-ых компонент ЛГМ; $c_{p 5}$ - теплоемкость газовой фазы $\rho_{5}$; $Q$ - массовая скорость возникновения / исчезновения газовой фазы; $\lambda_{T}$ и $D_{\mathrm{T}}-$ коэффициенты турбулентной теплопроводности и диффузии (для учета турбулентных процессов использован аналог теории Прандтля с постоянными числами Прандтля и Шмидта, обоснование и пределы применимости оговорены на стр. 247 и 312 в [Гришин, 1992]); через $R$ с нижним числовым индексом обозначаются массовые реакции возникновения (исчезновения) различных элементов, где $R_{1}$ соответствует образованию кислорода в реакции пиролиза, $R_{2}-$ испарению воды $\varphi_{2}$ из ЛГМ (сушка $\left.\varphi_{1}\right), R_{3}-$ исчезновению (горению) коксового остатка $\varphi_{3} ; s_{\sigma}$ - удельная поверхность коксика $\varphi_{3} ; R_{51}, R_{52}$ - массовые скорости образования кислорода $c_{1}$ и горючих газов $c_{2} ; \alpha_{c}-$ коксовое число сухого ЛГМ, $v_{\Gamma}-$ доля газообразных горючих продуктов пиролиза ЛГМ; $R_{5}$ - массовая скорость горения (окисления) горючих газов; $q_{2}, q_{3}$ и $q_{5}$ - количества выделяемой / поглощаемой энергии (тепловые эффекты) в процессах испарения $R_{2}$, горения 
конденсированного горючего $R_{3} \quad$ и $\quad$ газообразного горючего продукта пиролиза $R_{5}$ соответственно; $\alpha$ - коэффициент тепло- и газообмена, между атмосферой и моделируемым слоем ЛГМ; $\kappa_{R}-$ коэффициент радиационного излучения лесного массива; $\sigma-$ постоянная Стефана-Больцмана; $k_{01}, k_{02}, k_{03}$ и $E_{1}, E_{2}, E_{3}$ - предэкспоненты и энергии активации соответствующих физико-химических реакций $R_{1}, R_{2}, R_{3}$.

Существенной и неотъемлемой частью приведенной математической модели (1) - (11) является целый ряд коэффициентов и функций, характеризующих состав и пространственное распределение ЛГМ, а также скорости процессов сушки, пиролиза, горения и других. Выбор конкретных значений для оснастки модели производился на основе приведенных в литературных источниках справочных данных, а также результатах экспериментальных исследований [Гришин, 1992; Kuznetsov et al., 2019].

\section{Начальные и граничные условия}

Начальные распределения температуры $T$, объёмных долей $\varphi_{j}$ и массовых концентраций $c_{v}$ задаются во всей области моделирования. Обозначим границу этой области символом Г. В начальный момент (время моделирования $t=0$ ) весь лесной массив можно разбить на зону повышенной температуры, где происходят процессы пиролиза и горения, и две условные зоны $G_{-}$и $G_{+}$. $G_{-}$соответствует уже сгоревшим участкам леса, а $G_{+}$еще не затронутым пожаром областям, удаленным от очагов горения на такое расстояние, что эти подобласти характеризуются невозмущенными значениями $T_{\infty}, c_{1 \infty}$ и $c_{2 \infty}$. Взаимная геометрия указанных зон может быть весьма сложной, например, для многоочаговых пожаров. В очаге горения начальные распределения величин должны быть «самосогласованными», т. е. должно быть учтено наличие определённых связей физического характера между основными рассчитываемыми величинами. Вопросы задания «самосогласованных» начальных распределений в зоне пожара выходят за рамки данной статьи. Что касается подобластей «выжженная» $\mathrm{G}_{-}$и «невыжженная» $\mathrm{G}_{+}$, то начальные распределения в них задаются следующим образом:

$$
\begin{gathered}
\left.T\left(t, G_{-} \bigcup G_{+}\right)\right|_{t=0}=T_{\infty} ; \\
\left.c_{1}\left(t, G_{-} \bigcup G_{+}\right)\right|_{t=0}=c_{1 \infty},\left.\quad c_{2}\left(t, G_{-} \bigcup G_{+}\right)\right|_{t=0}=c_{2 \infty},\left.c_{3}\left(t, G_{-} \bigcup G_{+}\right)\right|_{t=0}=1-c_{1 \infty}-c_{2 \infty}, \\
\left.\varphi_{1}\left(t, G_{+}\right)\right|_{t=0}=\frac{\rho_{0}}{\rho_{1}},\left.\varphi_{2}\left(t, G_{+}\right)\right|_{t=0}=(1-\varsigma) W \frac{\rho_{0}}{\rho_{2}},\left.\quad \varphi_{3}\left(t, G_{+}\right)\right|_{t=0}=0, \quad \varphi_{4} \equiv 0, \\
\left.\varphi_{1}\left(t, G_{-}\right)\right|_{t=0}=0,\left.\quad \varphi_{2}\left(t, G_{-}\right)\right|_{t=0}=0,\left.\varphi_{3}\left(t, G_{-}\right)\right|_{t=0}=\alpha_{C} \frac{\rho_{0}}{\rho_{3}} .
\end{gathered}
$$

Здесь $\rho_{0}, W$ и $\varsigma$ - объёмная плотность, влагосодержание и зольность слоя ЛГМ соответственно, $\rho_{j}(\mathrm{j}=1,2,3,4)$ - плотности, соответствующие компонентам $\varphi_{j}$. Указанные распределения могут быть неоднородными в рассматриваемой области моделирования и задаются функциями от координат.

Граничные условия в моделируемой зоне Г задаются естественным образом:

$$
\left.\frac{\partial T}{\partial n}\right|_{\Gamma}=0,\left.\frac{\partial c_{1}}{\partial n}\right|_{\Gamma}=0,\left.\frac{\partial c_{2}}{\partial n}\right|_{\Gamma}=0 .
$$

\section{О методе численного решения, исходных данных и геометрии модельной задачи}

Записанная выше начально-краевая задача (1)-(16) решается численно с использованием системы Wolfram Mathematica [Barovik, Taranchuk, 2011a, b; Таранчук, Баровик, 2015]. Для аппроксимации применяются явные численные схемы с локально равномерной адаптируемой сеткой по пространству и переменным шагом по времени. Временные шаги выбираются из условий устойчивости численной схемы [Баровик и др., 
2013a, b] с учетом специфики и скоростей протекания физико-химических процессов на каждой конкретной итерации [Burger et al., 2020].

Рассмотрим модельную задачу распространения низового лесного пожара. Рассчитывается и анализируется динамика сопутствующих физико-химических процессов на площади в квадратной области 20 на 20 метров. Изучается процесс распространения пожара на участках, когда в центре области моделирования (в начале координат) «возникает» и начинает распространяться очаг горения. Для простоты описания считается, что направление ветра в пологе леса направлено по оси Ох (слева-направо на приведенных на рис. 1 графиках). При этом по направлению ветра, против ветра и перпендикулярно (на одном из флангов) имеются участки с отсутствием горючей растительности (поляны) [Taranchuk, Barovik, 2019]. Рассматриваются различные формы полян, а именно, круги, квадраты [Баровик, Таранчук, 2017] и прямоугольники.

На приведенных рисунках на заднем плане отображается плотность лесного горючего материала. Зеленым цветом показан незатронутый пожаром лес, а коричневым - участки с полным отсутствием горючего материала (поляны или уже сгоревший лес). На переднем плане рисунков цветовым градиентом отображаются карты распределения температуры в зоне горения.
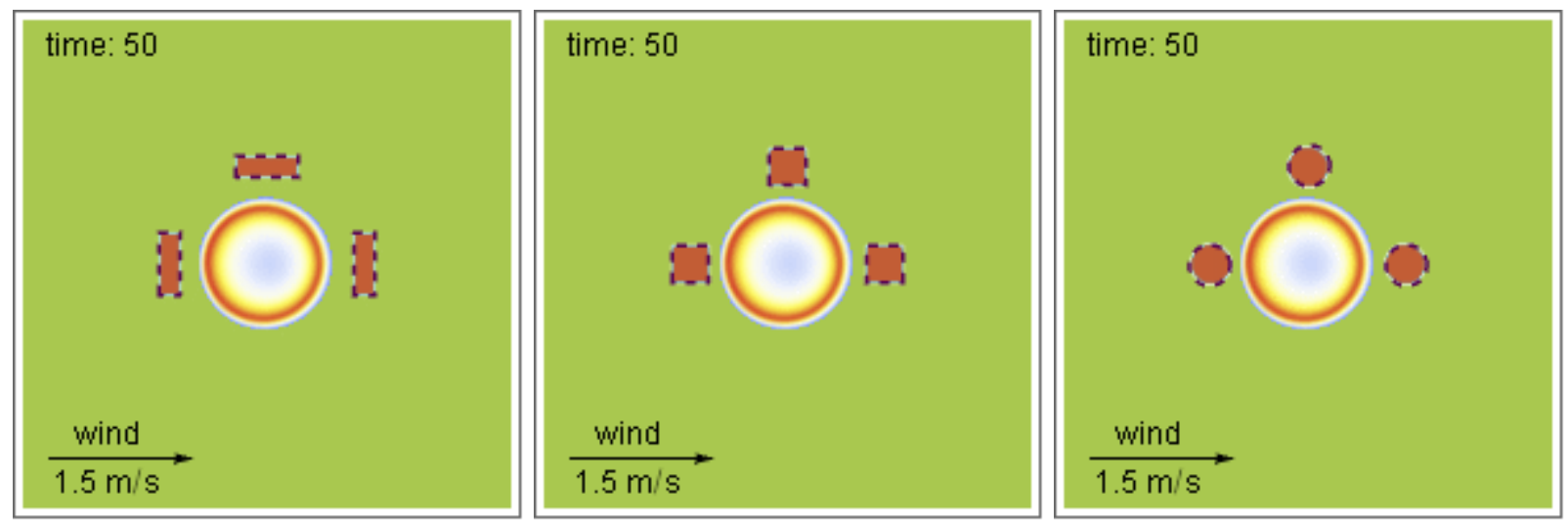

Рис. 1. Лесной пожар приближается к полянам различных форм

Fig. 1. Forest fire is approaching fuelbreaks of various shapes

Коэффициенты и определяющие параметры задачи, используемые при моделировании: температура окружающей среды $T_{\infty}=304 \mathrm{~K}$, высота слоя горючей растительности $\Delta h=0.25 \mathrm{M}$,

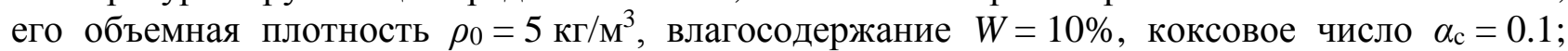
истинные плотности компонент ЛГМ $\rho_{1}=500 \mathrm{\kappa} / \mathrm{m}^{3}, \quad \rho_{2}=1000 \kappa \Gamma / \mathrm{M}^{3}, \quad \rho_{3}=200 \kappa \Gamma / \mathrm{M}^{3}$, $\rho_{4}=200 \kappa г / \mathrm{M}^{3} ; c_{1 \infty}=0.23, c_{2 \infty}=0, \rho_{\infty}=1.15 \kappa \Gamma / \mathrm{M}^{3} ;$ характеристики турбулентных процессов в газовой фазе $D_{\mathrm{T}}=1.5 \mathrm{~m}^{2} / \mathrm{c}, \quad \lambda_{T}=1000$ Дж/(м·c $\left.\cdot \mathrm{K}\right) ; \quad$ коэффициенты энергомассообмена $\kappa_{\mathrm{R}}=1.5 \mathrm{M}^{-1}, \quad \alpha=100 \mathrm{BT} /\left(\mathrm{M}^{2} \cdot \mathrm{K}\right) ;$ молекулярные массы $M_{1}=32, \quad M_{2}=28, \quad M_{3}=29, \quad M_{\mathrm{C}}=12$,

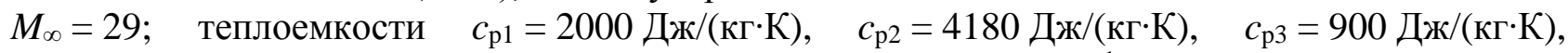

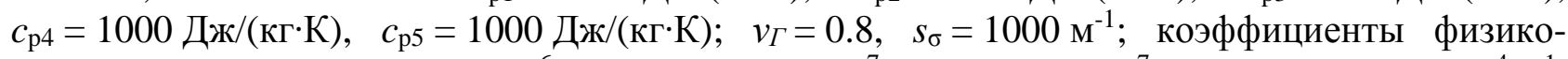
химических реакций $q_{2}=3 \cdot 10^{6}$ Дж/кг, $q_{3}=1.2 \cdot 10^{7}$ Дж/кг, $q_{5}=10^{7}$ Дж/кг, $k_{01}=3.63 \cdot 10^{4} \mathrm{c}^{-1}$, $k_{02}=6 \cdot 10^{5} \mathrm{~K}^{0.5} \mathrm{c}^{-1}, k_{03}=1000 \cdot \mathrm{c}^{-1}, E_{1} / R=9400 \mathrm{~K}, E_{2} / R=6000 \mathrm{~K}, E_{3} / R=10000 \mathrm{~K}$.

\section{Результаты моделирования преодоления пожаром полян различной формы}

На рис. 2-4 приводятся расчеты при равновесной скорости ветра на середине высоты пламени $V=1.5$ м/с. Показаны три формы полян: прямоугольники (см. рис. 2), квадраты (см. рис. 3) и круги (см. рис. 4). Геометрия отличается только формой полян, а площади полян и их расположения идентичны для всех трех расчетов. Также совпадают и приведенные на рисунках моменты времени для разных форм полян. 

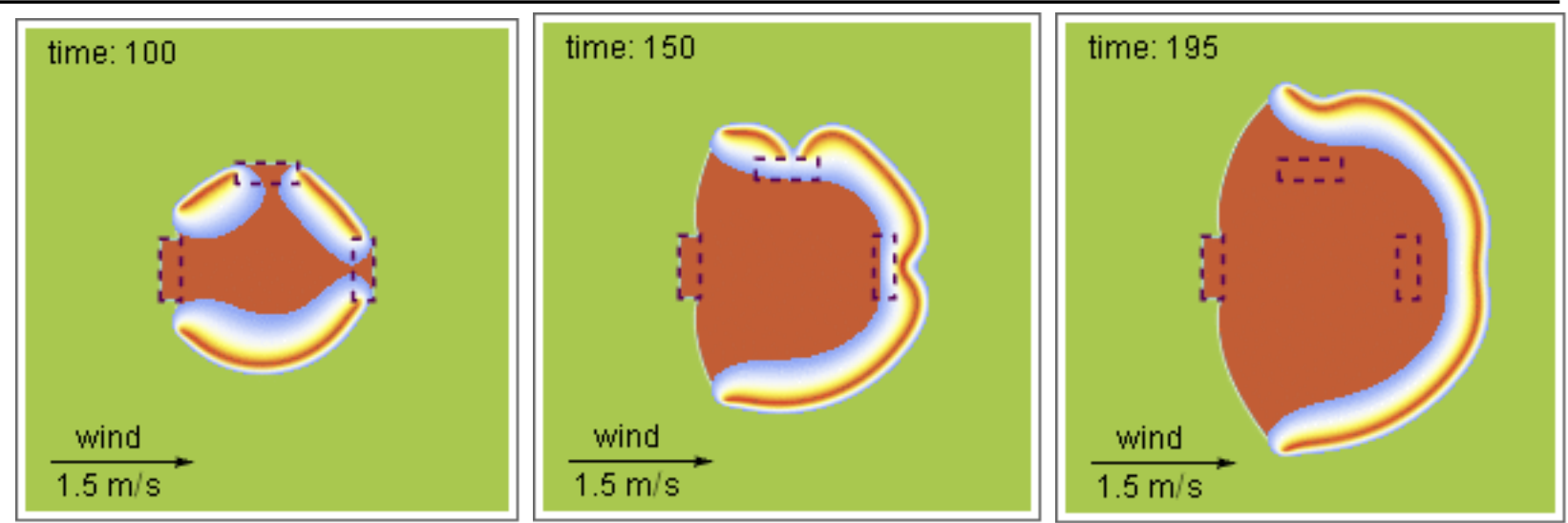

Рис. 2. Распространение пожара для случая полян прямоугольной формы

Fig. 2. Fire spread for the case of rectangular fuelbreaks
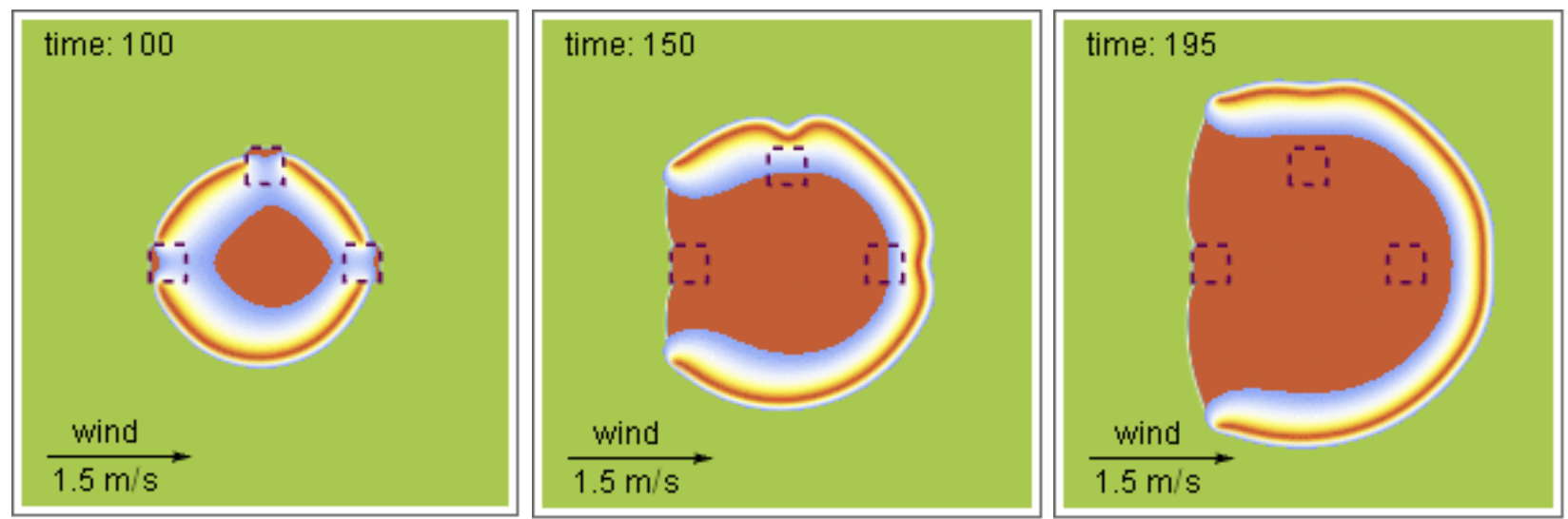

Рис. 3. Распространение пожара для случая полян квадратной формы

Fig. 3. Fire spread for the case of quadratic fuelbreaks
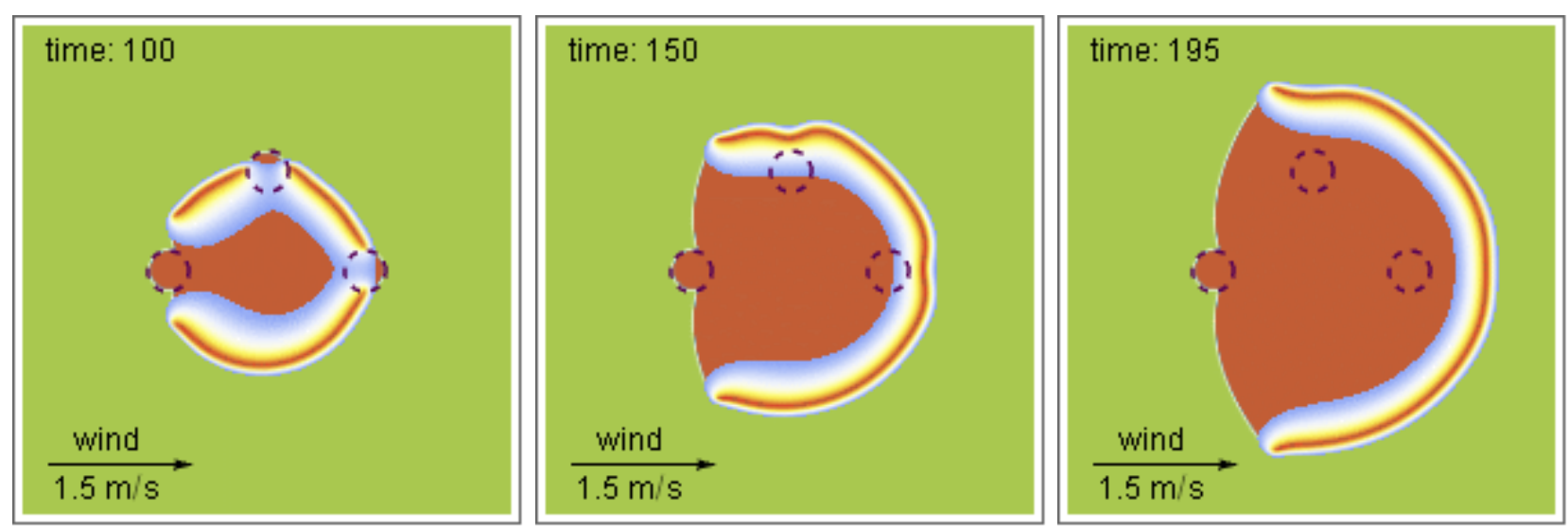

Рис. 4. Распространение пожара для случая полян в виде кругов при скорости ветра 1.5 м/с Fig. 4. Fire spread for the case of circular fuelbreaks at wind velocity $1.5 \mathrm{~m} / \mathrm{s}$

Качественно наблюдается следующее поведение. Сначала линия контура пожара разрывается, встретившись с полянами. Пожар «огибает» поляны. По направлению «против ветра» распространение пожара прекращается. А «по ветру» и «перпендикулярно» ветру разорванные контуры пожара вновь смыкаются и пожар распространяется единым фронтом. Заметно различие в результирующей конфигурации фронта после преодоления разных форм полян. 


\section{Учет влияния скорости ветра}

Рассмотрим влияние динамики лесного пожара при различных скоростях ветра [Таранчук, Баровик, 2017]. В данном случае будет рассматриваться случай наличия круглых полян. Выше (см. рис. 4) показан расчет для скорости ветра $V=1.5$ м/с.

На рис. 5 и 6 приведены два дополнительных расчета, отличающихся от расчета рис. 4 только тем, что скорости ветра $V$ равны 1 (один) и 2 (два) м/с соответственно. Особый интерес вызывает рис. 5. При слабых скоростях ветра пожар успешно преодолевают поляны во всех направлениях, в т. ч. и против ветра. Заметно и то, что ширина пожара во фронте более узкая, чем на флангах, а в тылу ширина пожара максимальна.
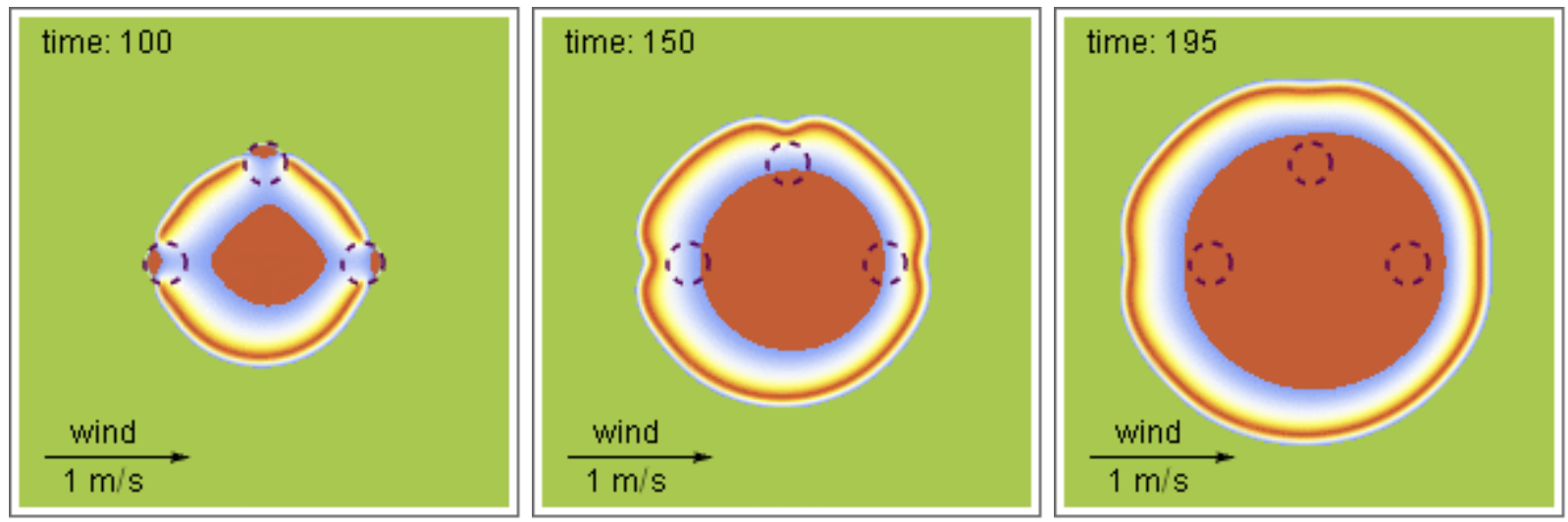

Рис. 5. Распространение пожара для случая полян в виде кругов при скорости ветра $1 \mathrm{~m} / \mathrm{c}$

Fig. 5. Fire spread for the case of circular fuelbreaks at wind velocity $1 \mathrm{~m} / \mathrm{s}$
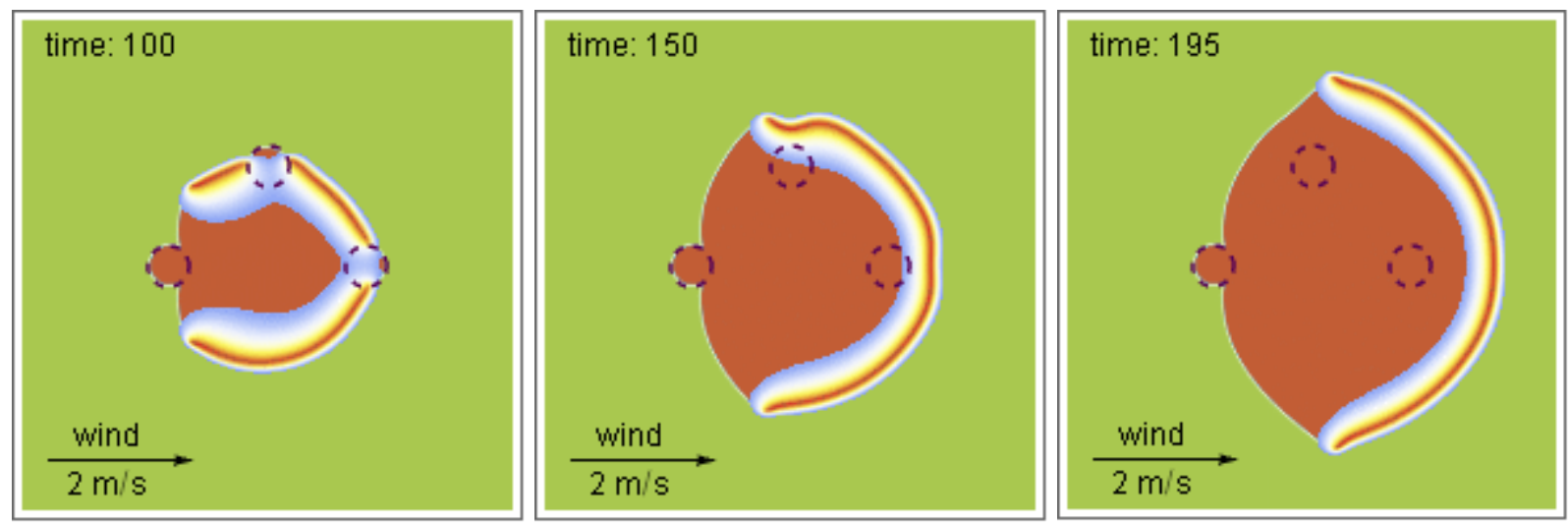

Рис. 6. Распространение пожара для случая полян в виде кругов при скорости ветра 2 м/с Fig. 5. Fire spread for the case of circular fuelbreaks at wind velocity $2 \mathrm{~m} / \mathrm{s}$

\section{Заключение}

На приведенных изображениях визуализированы только два (температура и объемная плотность сухих лесных горючих материалов) из шести рассчитываемых величин. По факту в модели вычисляются распределения величин, позволяющие оценить: появление и объем выделяемого водяного пара, выброс загрязняющих окружающую среду газов, образование угля и золы, расчет иных важных с практической точки зрения физико-химических процессов, влияющих на экологию [Таранчук, Баровик, 2014].

Нерешенным остается вопрос трудоемкости вычислений, невозможность использования модели в режиме реального времени. Разумным представляется подход, при котором результаты вычислительных экспериментов автоматически протоколируются в базах данных типовых сценариев развития процессов [Баровик, 2010], производится их интеллектуальная обработка [Таранчук, 2019], унификация, введение признаков и категорий, отнесение результатов серий расчетов лесных пожаров к конкретным территориям и климатическим условиям. В дальнейшем соответствующие каталоги могут использоваться в программных комплексах с полуэмпирическими моделями [Баровик и др., 2011, 2013с]. 


\section{Список литературы}

1. Барановский Н.В., Захаревич А.В. 2019. Физическое моделирование процессов зажигания еловой хвои углеродистой нагретой до высоких температур частицей. Вопросы лесной науки, 2 (1): 1-15. DOI: $10.31509 / 2658-607 x-2019-2-1-1-15$.

2. Баровик Д.В., Таранчук В.Б. 2017. Моделирование процессов распространения низовых лесных пожаров при наличии полян на пути огня. Математические методы в технике и технологиях ММТT, Том 12, Часть 1: 109-113.

3. Баровик Д.В., Таранчук В.Б. 2011с. Состояние проблемы и результаты компьютерного прогнозирования распространения лесных пожаров. Вестник БГУ. Серия 1, Физика. Математика. Информатика, 3: 78-84.

4. Баровик Д.В., Таранчук В.Б. 2011d. О развитии методики Ротермела и реализации двумерной компьютерной модели прогноза распространения лесных пожаров. Веснік Віцебскага дзяржаўнага універсітэта, 6 (66): 5-11.

5. Баровик Д.В., Таранчук В.Б. 2011е. Алгоритмические основы построения компьютерной модели прогноза распространения лесных пожаров. Вестник ПГУ. Серия С: Фундаментальные науки, 12: $51-56$.

6. Баровик Д.В., Таранчук В.Б. 2011f. Адаптация модели Ротермела для реализации в программном комплексе прогноза распространения лесных пожаров. Технологии техносферной безопасности, 6 (40): 6.

7. Баровик Д.В., Таранчук В.Б. 2010b. Об особенностях адаптации математических моделей вершинных верховых лесных пожаров. Вестник БГУ. Серия 1, Физика. Математика. Информатика, 1: $138-143$.

8. Баровик Д.В., Таранчук В.Б. 2010с. Численная реализация математической модели верховых лесных пожаров. Весці БДПУ. Серыя 3, 2 (64): 40-44.

9. Баровик Д.В. и др. 2013а. К обоснованию математических моделей низовых лесных пожаров. Труды института математики, 21 (1): 3-15.

10.Баровик Д.В. и др. 2013b. О корректности одной математической модели низовых лесных пожаров. Доклады Национальной академии наук Беларуси, 57 (4): 5-9.

11.Баровик Д.В. и др. 2013с. Структура и функционал модуля «оперативно-аналитический блок» программного комплекса регистрации и обработки сообщений о чрезвычайных ситуациях. Чрезвычайные ситуации: предупреждение и ликвидация, 2 (34): 84-94.

12.Баровик Д.В. и др. 2011. Методические и алгоритмические основы программного комплекса «Расчет и визуализация динамики лесного пожара». Чрезвычайные ситуации: предупреждение и ликвидация, 2 (30): 22-33.

13.Баровик Д.В. 2010. Базы данных результатов численного моделирования (на примере задачи распространения лесных пожаров). Вестник БГУ. Серия 1, Физика. Математика. Информатика, 2: 170-174.

14.Волокитина А.В. и др. 2020. Прогнозирование поведения пожаров растительности. Лесной журнал, 1: 9-25. DOI: 10.37482/0536-1036-2020-1-9-25.

15.Гладской И.Б. и др. 2019. К моделированию распространения природных пожаров с использованием ГИС-технологий. Экологический вестник центров Черноморского экономического сотрудничества, 16 (4): 13-21. DOI: 10.31429/vestnik-16-4-13-21.

16.Гоман П.Н. и др. 2011. Экспериментально-численное моделирование процесса горения и распространения огня в условиях лесного низового пожара. Технологии техносферной безопасности, 3 (37): 8.

17.Гришин А.М. 1992. Математическое моделирование лесных пожаров и новые способы борьбы с ними. Новосибирск: Наука, Сибирское отделение, 408.

18.Кулешов А.А., Мышецкая Е.Е. 2019. Результаты расчетов распространения фронта лесных пожаров по двумерной трехфазной модели. Препринты ИПМ им. М.В. Келдыша, 115: 1-9. DOI: $10.20948 /$ prepr-2019-115.

19.Ласута Г.Ф., Гоман П.Н. 2019. Моделирование процессов возникновения и распространения лесного низового пожара с оценкой уровня тепловой нагрузки от фронта пламени. Вестник Университета гражданской защиты МЧС Беларуси, 3 (2): 138-154. DOI: 10.33408/2519-237X.2019.3-2.138.

20.Марзаева В.И. 2019. Математическое моделирование распространения верховых лесных пожаров при наличии противопожарных разрывов и заслонов. Журнал технической физики, том 89, вып. 8: 1141-1149. DOI: 10.21883/JTF.2019.08.47883.392-18.

21.Чешко И.Д. и др. 2019. Экспертное исследование природных пожаров: Методическое пособие. СПб.: СПб университет ГПС МЧС России, 252. 
22.Сыродой С.В., Кузнецов Г.В. 2018. Влияние кинетической модели описания процессов термического разложения на результаты математического моделирования зажигания частиц древесной биомассы. Труды седьмой Российской национальной конференции по теплообмену. В $3 \mathrm{x}$ томах. М.: Издательский дом МЭИ: Том 1: 457-460.

23.Таранчук В.Б., Баровик Д.В. 2017. Методы, средства, отдельные результаты компьютерного моделирования низовых лесных пожаров. Информационные технологии и системы 2017 (ИТС 2017): материалы международной научной конференции. Минск: БГУИР: 178-179.

24.Таранчук В.Б., Баровик Д.В. 2015. О средствах Wolfram Mathematica для распараллеливания вычислений в компьютерных моделях лесных пожаров. Веб-программирование и интернеттехнологии WebConf 2015. Минск: Белорусский государственный университет: 108-109.

25.Таранчук В.Б., Баровик Д.В. 2014. Компьютерное моделирование лесных пожаров. Наука, инновации, инвестиции: сборник материалов 2-го Белорусско-Латвийского форума. Минск: Белорусский национальный технический университет: 73-75.

26.Таранчук В.Б. 2019. Методы и примеры интеллектуальной обработки данных для геологических моделей. Научные ведомости Белгородского государственного университета. Серия: Экономика. Информатика. 46 (3): 511-522. DOI 10.18413/2411-3808-2019-46-3-511-522.

27. Antonov D. et al. 2018. Experimental and Numerical Studies of Suppression of Forest Combustible Material Pyrolysis under Influence of Steam-Water Curtain. MATEC Web of Conferences: Heat and mass transfer in the thermal control system of technical and technological energy equipment, HMTTSC 2018, EDP Sciences: 01003.

28.Barovik D.V., Taranchuk V.B. 2011a. Crown Forest Fire Mathematical Model Realization in Wolfram Mathematica. Computer Algebra Systems in Teaching and Research, Vol. Mathematical Modeling in Physics, Civil Engineering, Economics and Finance: 5-15. ISBN: 978-83-930638-5-7.

29.Barovik D.V., Taranchuk V.B. 2011b. Results of Crown Forest Fires Mathematical Modelling. Computer Algebra Systems in Teaching and Research. Computer Algebra Systems in Teaching and Research, Vol. Mathematical Modeling in Physics, Civil Engineering, Economics and Finance: 16-22. ISBN: 978-83-930638-5-7.

30.Barovik D., Taranchuk V. 2010a. Mathematical modelling of running crown forest fires. Mathematical Modelling and Analysis, 15 (2): 161-174. DOI: 10.3846/1392-6292.2010.15.161-174.

31.Burger R. et al. 2020. Implicit-Explicit Methods for a Convection-Diffusion-Reaction Model of the Propagation of Forest Fires. Mathematics 2020, 8 (6): 1034. DOI: 10.3390/math8061034.

32.Dvornik A.A. et al. 2018. Potential threat to human health during forest fires in the Belarusian exclusion zone. Aerosol Science and Technology, 52 (8): 923-932. DOI: 10.1080/02786826.2018.1482408.

33.Frangieh N. et al. 2020. Wildfires front dynamics: 3D structures and intensity at small and large scales. Combustion and Flame, 211: 54-67. DOI: 10.1016/j.combustflame.2019.09.017.

34.Kuznetsov G.V. et al. 2019. Heat transfer and phase transformations in the localization of forest fuel combustion. Interfacial Phenomena and Heat Transfer, 7 (2): 167-195. DOI: 10.1615/InterfacPhenom HeatTransfer.2019031564.

35.Perminov V., Goudov A. 2017. Mathematical modeling of forest fires initiation, spread and impact on environment. International Journal of GEOMATE, 13 (35): 93-99. DOI: 10.21660/2017.35.6704.

36. Taranchuk V., Barovik D. 2019. Numerical Modelling of Surface Forest Fire Spread in Nonuniform Woodland. Computer Algebra Systems in Teaching and Research (CASTR 2019), Vol. VIII: 159 - 168. ISBN: 978-83-7051-956-8.

37.theguardian.com. 2020. Economic impact of Australia's bushfires set to exceed $\$ 4.4 \mathrm{bn}$ cost of Black Saturday. Available at: www.theguardian.com/australia-news/2020/jan/08/economic-impact-ofaustralias-bushfires-set-to-exceed-44bn-cost-of-black-saturday (accessed 1 July 2020).

38.wwf.org.ua. 2020. Statement from WWF-Australia on Australia's bushfire emergency. Available at: www.wwf.org.au/news/news/2020/statement-from-wwf-australia-on-australia-s-bushfire-emergency (accessed 1 July 2020).

\section{References}

1. Antonov D. et al. 2018. Experimental and Numerical Studies of Suppression of Forest Combustible Material Pyrolysis under Influence of Steam-Water Curtain. MATEC Web of Conferences: Heat and mass transfer in the thermal control system of technical and technological energy equipment, HMTTSC 2018, EDP Sciences: 01003. 
2. Barovik D.V., Taranchuk V.B. 2011a. Crown Forest Fire Mathematical Model Realization in Wolfram Mathematica. Computer Algebra Systems in Teaching and Research, Vol. Mathematical Modeling in Physics, Civil Engineering, Economics and Finance: 5-15. ISBN: 978-83-930638-5-7.

3. Barovik D.V., Taranchuk V.B. 2011b. Results of Crown Forest Fires Mathematical Modelling. Computer Algebra Systems in Teaching and Research. Computer Algebra Systems in Teaching and Research, Vol. Mathematical Modeling in Physics, Civil Engineering, Economics and Finance: 16-22. ISBN: 978-83930638-5-7.

4. Barovik D., Taranchuk V. 2010a. Mathematical modelling of running crown forest fires. Mathematical Modelling and Analysis, 15 (2): 161-174. DOI: 10.3846/1392-6292.2010.15.161-174.

5. Burger R. et al. 2020. Implicit-Explicit Methods for a Convection-Diffusion-Reaction Model of the Propagation of Forest Fires. Mathematics 2020, 8 (6): 1034. DOI: 10.3390/math8061034.

6. Dvornik A.A. et al. 2018. Potential threat to human health during forest fires in the Belarusian exclusion zone. Aerosol Science and Technology, 52 (8): 923-932. DOI: 10.1080/02786826.2018.1482408.

7. Frangieh N. et al. 2020. Wildfires front dynamics: 3D structures and intensity at small and large scales. Combustion and Flame, 211: 54-67. DOI: 10.1016/j.combustflame.2019.09.017.

8. Kuznetsov G.V. et al. 2019. Heat transfer and phase transformations in the localization of forest fuel combustion. Interfacial Phenomena and Heat Transfer, 7 (2): 167-195. DOI: 10.1615/InterfacPhenom HeatTransfer.2019031564.

9. Perminov V., Goudov A. 2017. Mathematical modeling of forest fires initiation, spread and impact on environment. International Journal of GEOMATE, 13 (35): 93-99. DOI: 10.21660/2017.35.6704.

10. Taranchuk V., Barovik D. 2019. Numerical Modelling of Surface Forest Fire Spread in Nonuniform Woodland. Computer Algebra Systems in Teaching and Research (CASTR 2019), Vol. VIII: 159-168. ISBN: 978-83-7051-956-8.

11. theguardian.com. 2020. Economic impact of Australia's bushfires set to exceed $\$ 4.4 \mathrm{bn}$ cost of Black Saturday. Available at: www.theguardian.com/australia-news/2020/jan/08/economic-impact-ofaustralias-bushfires-set-to-exceed-44bn-cost-of-black-saturday (accessed 1 July 2020).

12. wwf.org.ua. 2020. Statement from WWF-Australia on Australia's bushfire emergency. Available at: www.wwf.org.au/news/news/2020/statement-from-wwf-australia-on-australia-s-bushfire-emergency (accessed 1 July 2020).

13. Baranovskiy N.V., Zakharevich A.V. 2019. Experimental modelling of spruce needles ignition by the carbonaceous heated up to high temperatures particle. Forest science issues, 2 (1): 1-15 (in Russian). DOI: $10.31509 / 2658-607 x-2019-2-1-1-15$.

14. Barovik D.V., Taranchuk V.B. 2017. Modelirovanie protsessov rasprostraneniya nizovykh lesnykh pozharov pri nalichii polyan na puti ognya [Modeling of ground forest fires spread processes in case of fuelbreaks presence]. Matematicheskie metody v tekhnike i tekhnologiyakh - MMTT [Mathematical methods in engineering and technology - MMET], Vol 12, Part 1: 109-113.

15. Barovik D.V., Taranchuk V.B. 2011c. Sostoyanie problemy i rezul'taty komp'yuternogo prognozirovaniya rasprostraneniya lesnykh pozharov [State of the problem and results of computer forecasting of the spread of forest fires]. Vestnik BGU. Seriya 1, Fizika. Matematika. Informatika [BSU Bulletin. Series 1, Physics. Mathematics. Computer Science], 3: 78-84.

16. Barovik D.V., Taranchuk V.B. 2011d. On Rothermel's model improvement and implementation of two-dimensional computer model of forest fire spread prediction. Vesnik Vitsebskaga dzyarzhay̆naga universiteta, 6 (66): 5-11 (in Russian).

17. Barovik D.V., Taranchuk V.B. 2011e. Algorithmic fundamentals of computer model for forest fires prediction. Vestnik PGU. Seriya C: Fundamental'nye nauki, 12: 51-56 (in Russian).

18. Barovik D.V., Taranchuk V.B. 2011f. Rothermel's model adaptation for implementation in forest fires forecast software. Tekhnologii tekhnosfernoy bezopasnosti, 6 (40): 6 (in Russian).

19. Barovik D.V., Taranchuk V.B. 2010b. Ob osobennostyakh adaptatsii matematicheskikh modeley vershinnykh verkhovykh lesnykh pozharov [The adaptation of the features of mathematical models of top crown forest fire]. Vestnik BGU. Seriya 1, Fizika, Matematika, Informatika [BSU Bulletin. Series 1, Physics. Mathematics. Computer Science], 1: 138-143.

20. Barovik D.V., Taranchuk V.B. 2010c. Chislennaya realizatsiya matematicheskoy modeli verkhovykh lesnykh pozharov [Numerical implementation of mathematical models of upland forest fires]. Vestsi BDPU. Seryya 3 [BSPU News. Series 3], 2 (64): 40-44 (in Russian).

21. Barovik D.V. et al. 2013a. Methods of forest fires computer modelling, 21 (1): 3-15 (in Russian).

22. Barovik D.V. et al. 2013b. On the correctness of a mathematical model of ground forest fires. Doklady Natsional'noy akademii nauk Belarusi, 57 (4): 5-9 (in Russian). 
23. Barovik D.V. et al. 2013c. Struktura i funktsional modulya "operativno-analiticheskiy blok" programmnogo kompleksa registratsii i obrabotki soobshcheniy o chrezvychaynykh situatsiyakh [The structure and functionality of the module "operational analysis unit" software package for recording and processing of emergency messages]. Chrezvychaynye situatsii: preduprezhdenie i likvidatsiya [Emergency situations: prevention and elimination], 2 (34): 84-94.

24. Barovik D.V. et al. 2011. Metodicheskie i algoritmicheskie osnovy programmnogo kompleksa "Raschet i vizualizatsiya dinamiki lesnogo pozhara" [Methodological and algorithmic foundations of software "Calculation and visualization of the dynamics of forest fires"]. Chrezvychaynye situatsii: preduprezhdenie i likvidatsiya [Emergency situations: prevention and elimination], 2 (30): 22-33.

25. Barovik D.V. 2010. Bazy dannykh rezul'tatov chislennogo modelirovaniya (na primere zadachi rasprostraneniya lesnykh pozharov) [Databases numerical simulation results (for example, the problem of propagation of forest fires)]. Vestnik BGU. Seriya 1, Fizika. Matematika. Informatika [BSU Bulletin. Series 1, Physics. Mathematics. Computer Science], 2: 170-174.

26. Volokitina A.V. et al. 2020. Vegetation fire behavior prediction. Lesnoy zhurnal, 1: 9-25 (in Russian). DOI: 10.37482/0536-1036-2020-1-9-25.

27. Gladskoy I.B. et al. 2019. To modeling the spread of forest fires using GIS technologies. Ecological Bulletin of Research Centers of the Black Sea Economic Cooperation, 16 (4): 13-21 (in Russian). DOI: $10.31429 /$ vestnik-16-4-13-21

28. Goman P.N. et al. 2011. Experimental and numerical simulation of combustion as well as the spread of fire in the forest groundfire. Tekhnologii tekhnosfernoy bezopasnosti, 3 (37): 8 (in Russian).

29. Grishin A.M. 1997. Mathematical modeling of forest fires and new methods of fighting them. Ed. by Albini F. Tomsk, Russia: Tomsk State University, 390.

30. Kuleshov A.A., Myshetskaya E.E. 2019. Results of computation of the forest fires front propagation based on a twodimensional three-phase model. KIAM Preprint, 115: 1-9 (in Russian). DOI: 10.20948/prepr-2019-115.

31. Lasuta G.F., Goman P.N. 2019. Modeling of the processes of the occurrence and spread of forest groundfire with the estimation of the level of flame front heat load. Journal of Civil Protection, 3 (2): 138-154 (in Russian). DOI: 10.33408/2519-237X.2019.3-2.138.

32. Marzaeva V.I. 2019. Mathematical Modeling of Canopy Forest Fire Spread in the Presence of Fire Breaks and Barriers. Technical Physics, volume 64: 1073-1081. DOI: 10.21883/JTF.2019.08.47883.392-18.

33. Cheshko I.D. et al. 2019. Ekspertnoe issledovanie prirodnykh pozharov: Metodicheskoe posobie [Expert research wildfires: Methodological guide]. SPb.: SPb universitet GPS MChS Rossii, 252.

34. Syrodoy S.V., Kuznetsov G.V. 2018. Vliyanie kineticheskoy modeli opisaniya protsessov termicheskogo razlozheniya na rezul'taty matematicheskogo modelirovaniya zazhiganiya chastits drevesnoy biomassy [Effect of kinetic model descriptions of the processes of thermal decomposition of mathematical modeling ignition woody biomass particles]. Trudy sed'moy Rossiyskoy natsional'noy konferentsii po teploobmenu [Proceedings of the Seventh Russian National Heat Transfer Conference]. M.: Izdatel'skiy dom MEI: Vol 1: 457-460.

35. Taranchuk V.B., Barovik D.V. 2017. Metody, sredstva, otdel'nye rezul'taty komp'yuternogo modelirovaniya nizovykh lesnykh pozharov [Methods, tools, individual results of computer modeling of local forest fires.] Informatsionnye tekhnologii i sistemy 2017 (ITS 2017): materialy mezhdunarodnoy nauchnoy konferentsii [Information Technologies and Systems 2017 (ITS 2017): Proceedings of the International Scientific Conference]. Minsk: BGUIR: 178-179.

36. Taranchuk V.B., Barovik D.V. 2015. O sredstvakh Wolfram Mathematica dlya rasparallelivaniya vychisleniy v komp'yuternykh modelyakh lesnykh pozharov About Wolfram Mathematica tools for parallel computing in computer models of forest fires]. Veb-programmirovanie i internet-tekhnologii WebConf 2015 [Web Development and Internet Technologies WebConf 2015]. Minsk: Belorusskiy gosudarstvennyy universitet: 108-109.

37. Taranchuk V.B., Barovik D.V. 2014. Komp'yuternoe modelirovanie lesnykh pozharov [Computer simulation of forest fires]. Nauka, innovatsii, investitsii: sbornik materialov 2-go Belorussko-Latviyskogo foruma. [Science, innovations, investments: collection of materials of the 2nd Belarusian-Latvian Forum]. Minsk: Belorusskiy natsional'nyy tekhnicheskiy universitet: 73-75.

38. Taranchuk V.B. 2019. Methods and examples of intelligent data processing for geological models. Belgorod State University Scientific Bulletin. Economics. Information technologies. 46 (3): 511-522 (in Russian). DOI 10.18413/2411-3808-2019-46-3-511-522. 


\section{ИНФОРМАЦИЯ ОБ АВТОРАХ}

Таранчук Валерий Борисович, доктор физикоматематических наук, профессор, профессор кафедры компьютерных технологий и систем факультета прикладной математики и информатики Белорусского государственного университета, г. Минск, Беларусь

Баровик Дмитрий Валентинович, кандидат физико-математических наук, доцент кафедры компьютерных технологий и систем факультета прикладной математики и информатики Белорусского государственного университета, г. Минск, Беларусь

\section{INFORMATION ABOUT THE AUTHORS}

Valery B. Taranchuk, Doctor of Physical and Mathematical Sciences, Professor, Professor of the Department of Computer Technologies and Systems, Faculty of Applied Mathematics and Informatics, Belarusian State University, Minsk, Belarus

Dmitry V. Barovik, Candidate of Physical and Mathematical Sciences, Associate Professor of the Department of Computer Technologies and Systems, Faculty of Applied Mathematics and Informatics, Belarusian State University, Minsk, Belarus 